\title{
Enhanced thermal conductivity of graphene nanoplatelets epoxy composites
}

\author{
LukASZ JAROSINSKI ${ }^{1}$, ANDRZEJ RYBAK ${ }^{2}$, KAROLINA GASKA ${ }^{1,3, *}$, GRZEGORZ KMITA ${ }^{2}$, \\ RENATA POREBSKA ${ }^{2}$, CZESLAW KAPUSTA ${ }^{1}$ \\ ${ }^{1}$ Department of Solid State Physics, Faculty of Physics and Applied Computer Science, \\ AGH University of Science and Technology, Krakow, Poland \\ ${ }^{2}$ ABB Corporate Research Center, Krakow, Poland \\ ${ }^{3}$ Department of Materials and Manufacturing Technology, High Voltage Engineering Division, \\ Chalmers University of Technology, Gothenburg, Sweden
}

\begin{abstract}
Efficient heat dissipation from modern electronic devices is a key issue for their proper performance. An important role in the assembly of electronic devices is played by polymers, due to their simple application and easiness of processing. The thermal conductivity of pure polymers is relatively low and addition of thermally conductive particles into polymer matrix is the method to enhance the overall thermal conductivity of the composite. The aim of the presented work is to examine a possibility of increasing the thermal conductivity of the filled epoxy resin systems, applicable for electrical insulation, by the use of composites filled with graphene nanoplatelets. It is remarkable that the addition of only $4 \mathrm{wt} \%$ of graphene could lead to $132 \%$ increase in thermal conductivity. In this study, several new aspects of graphene composites such as sedimentation effects or temperature dependence of thermal conductivity have been presented. The thermal conductivity results were also compared with the newest model. The obtained results show potential for application of the graphene nanocomposites for electrical insulation with enhanced thermal conductivity. This paper also presents and discusses the unique temperature dependencies of thermal conductivity in a wide temperature range, significant for full understanding thermal transport mechanisms.
\end{abstract}

Keywords: graphene nanoplatelets; thermal conductivity; electrical resistivity, epoxy resin, high-shear exfoliation

\section{Introduction}

The thermal properties of materials have recently attracted a lot of attention, which is driven by the need for effective heat removal in systems such as CMOS, LEDs, automotive products and power electronics [1-3]. The continuing miniaturization of microelectronic devices is setting higher requirements for reliability, performance, and processing techniques for advanced packaging materials [4]. The packaging materials are required to possess the functionally desired physical and mechanical properties, including electrical insulation, and to be lightweight and easily processed. Polymers have been mainly used as packaging materials for encapsulating integrated electronic circuits because of their excellent characteristics. However, the thermal conductivity of polymers is relatively low

*E-mail: kgaska@agh.edu.pl (of the order of $0.2 \mathrm{~W} \cdot \mathrm{m}^{-1} \cdot \mathrm{K}^{-1}$ for epoxy resin) therefore, improving their thermal conductivity is a crucial issue to be solved [5]. The traditional method is to introduce ceramic particles with high thermal conductivity into the matrix in order to increase their effective thermal conductivity. As an example, for a silica-epoxy composite with 31 vol.\% of filler content the achieved effective thermal conductivity was $0.61 \mathrm{~W} \cdot \mathrm{m}^{-1} \cdot \mathrm{K}^{-1}$, whereas the usage of high thermally conductive boron nitride with 5 vol.\% content can result in $0.5 \mathrm{~W} \cdot \mathrm{m}^{-1} \cdot \mathrm{K}^{-1}$ [6]. However, achieving a high thermal conductivity generally requires a very high loading content of ceramic fillers (>40 vol.\%), which can cause the mechanical properties deterioration and processing difficulties of the composite with increased viscosity $[6,7]$.

In the last decades, polymer nanocomposites with carbon based nanofillers have been 
widely studied in order to develop materials with multifunctional properties such as high mechanical performance, heat dissipation, damage sensing, electrostatic discharge, large operating temperature range and chemical resistance [8, 9]. Lately, especially graphene nanoplatelets ( $\mathrm{GnPs}$ ) have attracted a great attention, and have been used as a filler for reinforcement and functionalization of polymers [10-16]. Such great interest appeared due to graphene unique properties. For instance, two dimensional (2D) graphene flakes (GFs) have drawn an interest due to their extremely high thermal conductivity [17]. Namely, the thermal conductivity of single-layer graphene can reach about $5000 \mathrm{~W} \cdot \mathrm{m}^{-1} \cdot \mathrm{K}^{-1}[18,19]$, while the thermal conductivity of few-layer graphene is lower [20], for example, in case of four layers, it is about $2000 \mathrm{~W} \cdot \mathrm{m}^{-1} \cdot \mathrm{K}^{-1}[20]$. Although graphene has extremely high thermal conductivity, it possesses also high electrical conductivity which should be also taken into account. Thus, it remains a huge technical challenge to prepare electrically insulating polymer composites with high thermal conductivity, and additionally without sedimentation effect.

Therefore, the aim of the presented work was to enhance the thermal conductivity of graphene polymer composites with preserved electrical insulation properties and homogeneous dispersion of the filler. Such composites belong to an important class of materials for applications in microelectronics. The obtained results show potential for application of the graphene nanocomposites for electrical insulation with enhanced thermal conductivity. One can find a lot of papers on graphene composite, however, the results presented in this article are attractive from the application point of view since the possibility to maintain the insulating properties is indicated. Moreover, a method for measuring the sedimentation effects is shown. In addition, interesting results of thermal conductivity temperature dependences in a wide range of temperatures have been presented. The experimental results are in a good agreement with the recently developed theoretical model for the analysis of thermal conductivity of isotropic composites containing randomly embedded GnPs [16].

\section{Experimental}

\subsection{Materials}

Graphene nanoplatelets powder AO-3 supplied by Graphene Supermarket was used as a filler. The average particle size was $4.5 \mu \mathrm{m}$ and the average thickness of these non-functionalized nanopowder particles was $12 \mathrm{~nm}$. The properties of used GnPs were presented in Table 1.

Table 1. Properties of used GnPs, available from the product data sheet.

\begin{tabular}{lc}
\hline \multicolumn{2}{c}{ Specifications } \\
\hline \hline Average flake thickness & $12 \mathrm{~nm}$ \\
Average particle size & $4.5 \mu \mathrm{m}(1.5 \mu \mathrm{m}$ to $10 \mu \mathrm{m})$ \\
Density & $2.25 \mathrm{~g} \cdot \mathrm{cm}^{-3}$ \\
Specific surface area & $80 \mathrm{~m}^{2} \cdot \mathrm{g}^{-1}$ \\
\hline
\end{tabular}

Epoxy resin derived from bisphenol A (DGEBA) CY 228-1 hardened with anhydride HY918 produced by Huntsman was used. This system forms a liquid resin with low viscosity and requires heating to start cross-linking. This system was chosen due to its very good insulating and mechanical properties, relatively good performance and easiness of processing. A flexibilizer DY 045 has been used to improve the mechanical properties. Additionally, a small amount of an amine accelerator DY 062 has been used in order to catalyze the curing process. Thermal conductivity of neat epoxy resin was $0.19 \mathrm{~W} \cdot \mathrm{m}^{-1} \cdot \mathrm{K}^{-1}$.

\subsection{Composites preparation}

First step in composite preparation was a liquid phase exfoliation of GnP particles by means of high shear mixer also called rotor-stator mixer. Highshear mixer leads to creation of high shear forces, particles collisions and jet cavitation which causes efficient exfoliation of graphene nanoplatelets [21, 22]. It was recently reported, that rapid, turbulent fluid flow and strong particles interactions with each other and with stator walls are sufficient to overcome van der Walls forces leading to production high quality graphene [23]. What is more, the exfoliation efficiency is higher than liquid sonication or ball milling exfoliation methods and can 


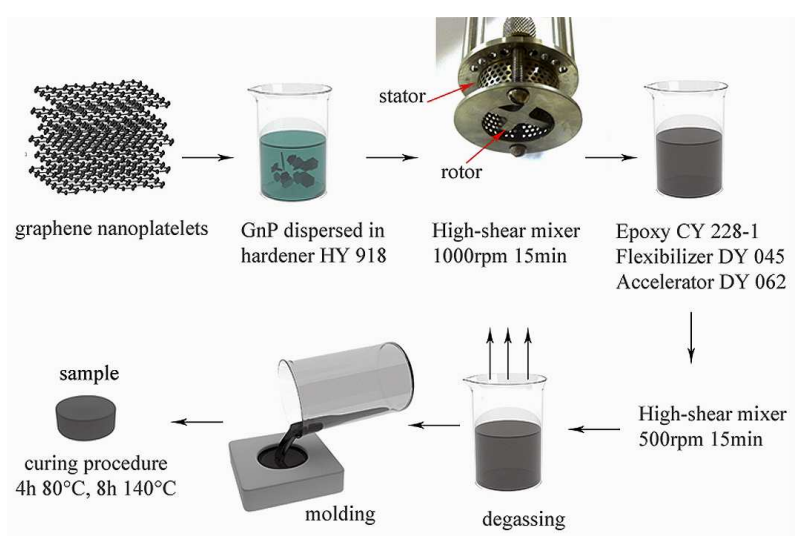

Fig. 1. Fabrication process of the polymer composites with the GnPs.

be treated as scalable method of graphene production [22, 24]. Firstly, GnP powder was mixed at $1000 \mathrm{rpm}$ for $15 \mathrm{~min}$ with a very low viscosity hardener HY 918 by means of Silverson L5M-A equipped with Emulsor Screen (Fig. 1). After this step, a stable suspension of exfoliated graphene nanoplatelets in the hardener was obtained. Thereafter, an epoxy resin CY 228-1, flexibilizer DY 045 and accelerator DY 062 were added. The specimens were mixed again at $500 \mathrm{rpm}$ for $15 \mathrm{~min}$ in order to achieve a homogeneous dispersion of GnPs. The composites were carefully degassed for one hour to avoid the formation of pores. Afterwards, the specimens were casted in a silicon mold with appropriate shape, which depended on the requirements for electrical and thermal conductivity measurements (more in the characterization section). The curing process was performed at $80^{\circ} \mathrm{C}$ for $4 \mathrm{~h}$, then $140{ }^{\circ} \mathrm{C}$ for $8 \mathrm{~h}$. The detailed experimental scheme of the processing of the epoxy composite is shown in Fig. 1. The composite specimens were prepared by filling epoxy with graphene at 0.1 wt. $\%, 0.5$ wt. $\%, 1.0$ wt. $\%, 2.0$ wt. $\%$ and $4.0 \mathrm{wt} . \%$ content, respectively.

\subsection{Characterization}

The electrical resistivity of graphene-epoxy composites was measured with a Keithley High Resistance Meter 6517B. Cylindrically shaped samples with a diameter of $35 \mathrm{~mm}$ and a thickness of $1 \mathrm{~mm}$ to $2 \mathrm{~mm}$ were used for the electrical resistivity measurements. Electrical resistivity measurements were performed at room temperature using two methods: a four-point probe (at a voltage of $50 \mathrm{~V}$ ) and in sandwich configuration (at a voltage of $1 \mathrm{kV}$ ) with a Faraday cage in order to avoid the influence of electromagnetic field. The thermal conductivity was investigated by transient heat source (Hot Disc Thermal Constant Analyzer TPS 500) technique (Fig. 2a). The measurements were carried out according to ISO 22007-2:2008 test method for determination of thermal properties. The sensor (Kapton $6.394 \mathrm{~mm}$ ) was sandwiched between two identical disks with a diameter of $35 \mathrm{~mm}$ and with a thickness of $10 \mathrm{~mm}$ to $15 \mathrm{~mm}$. A constant power of $0.8 \mathrm{~W}$ was applied for $40 \mathrm{~s}$ and the generated heat dissipated within the double spiral was conducted into the surroundings, causing a rise in the temperature of the sensor and the samples. This system allowed the study of homogeneity of filler (sedimentation effect) in the composite.
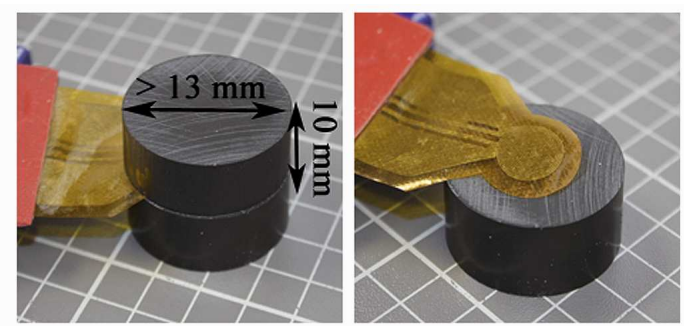

(a)

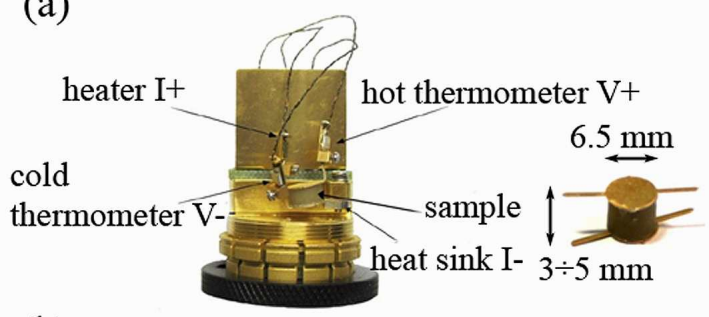

(b)

Fig. 2. Sample arrangement used for thermal conductivity measurement (a) Hot Disc TPS 500; (b) PPMS by Quantum Design, thermal transport option.

Temperature dependence of thermal conductivity has been studied by Physical Property Measurement System (PPMS) with a thermal transport 
option (TTO) by Quantum Design. PPMS is an instrument where the thermal conductivity is measured on small samples by the linear flow method at temperatures in the range of $300 \mathrm{~K}$ to liquid helium temperatures $2 \mathrm{~K}$. The sample, cooler, heater and thermometers were placed in an evacuated chamber in order to minimize the heat losses. At a given power setting of the heater, the temperature difference between the two sensors was used to calculate the thermal conductivity. The composite samples of a cylindrical shape with a diameter of $5 \mathrm{~mm}$ and a thickness of $3 \mathrm{~mm}$ to $4 \mathrm{~mm}$ were mounted between the gold plated cooper electrodes and fixed with electrically and thermally conductive epoxy bound (Fig. 2b.).

The nanostructure of the samples was investigated by TEM-transmission electron microscopy (FEI TECNAI TF 20 (FEG) $200 \mathrm{kV}$ ). The samples were prepared using FIB-FEI Quanta 3D 200i DualBeam.

\section{Results and discussion}

\subsection{Structure of the samples}

Fig. 3 shows TEM images of $\mathrm{GnP}$ in epoxy matrix. The images were made for the composite containing $1 \mathrm{wt} . \%$ of the filler. One can observe graphene flakes stacked in few-layer structure. Probably, during the curing process, Van der Waals forces caused graphene flakes to agglomerate which could deteriorate the properties of individual graphene flakes. However, it can be seen that the flakes are delaminated and have a crumpled surface which proves that the chosen exfoliation technique has an influence on graphene structure.

\subsection{Thermal and electrical properties of the GnP-epoxy composites}

Fig. 4 shows thermal conductivity and electrical resistivity of the samples versus graphene content. It is evident that the thermal conductivity increases steadily with increasing filler content. The measurement uncertainties were not taken into account, because they were smaller than the points on the graph. The effective heat conduction of the composite depends on

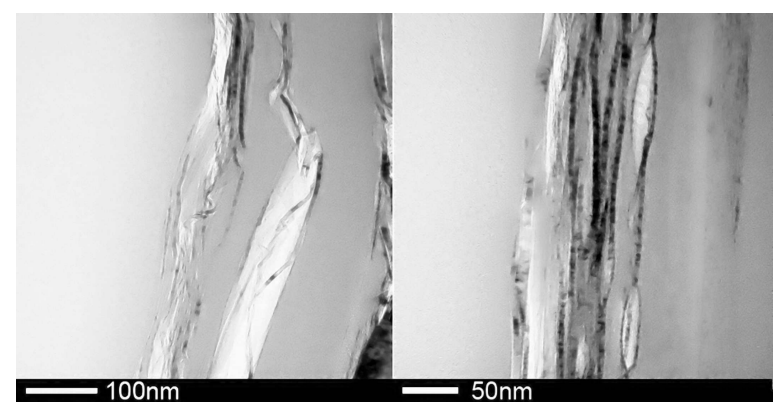

Fig. 3. TEM images for composite with 1 wt. $\%$ of graphene.

the thermal resistance between GnPs and the matrix and scattering processes on its interface [25]. Heat propagation in GnP-epoxy composite is generated mainly due to acoustic phonons and electrons scattering processes. The increasing trend promises higher thermal conductivity at larger $\mathrm{GnPs}$ concentration. It is worth to note that a high thermal conductivity enhancement of $132 \%$ was achieved for the composite with only $4 \mathrm{wt} . \%$ of graphene and $68 \%$ increase for 2 wt. $\%$ of the filler. For comparison Wang et al. [16] obtained slight enhancement of thermal conductivity for 3 wt. $\%$ of GnPs, however, for $5 \mathrm{wt} . \%$ of expanded GnPs $115 \%$ increase was observed. One can see that application of graphene with bigger particle size $(25 \mu \mathrm{m})$ performs better, showing significant improvement of thermal conductivity compared to epoxy filler with smaller $5 \mu \mathrm{m} \mathrm{GnP} \mathrm{[5].} \mathrm{For} \mathrm{larger}$ nanofiller particles it is easier to form a conduction path, which increases the thermal conductivity of the nanocomposites. Though the conductive paths can enhance electrical conductivity, but the main aim of this work is to maintain the insulating properties of the composites. Therefore, graphene with smaller particle size $(4.5 \mu \mathrm{m})$ has been applied to avoid a percolation threshold in resistivity. One can see that the obtained nanocomposites possess not only enhanced thermal conductivity but also high electrical resistivity, which shows their possible application in electronics [26, 27]. It is worth to note that the produced composites are electrical insulators which was the assumption of this study. For instance, the composite with 4 wt.\% of graphene has a four-point resistivity of about 
$8 \times 10^{10} \Omega \cdot \mathrm{cm}$. At larger $\mathrm{GnP}$ concentrations, percolation threshold for electrical conductivity was expected [28]. Up to this point, composites retain their insulating properties improving simultaneously their thermal properties. The obtained results show potential for application of the graphene nanocomposites for electrical insulation with enhanced thermal conductivity.

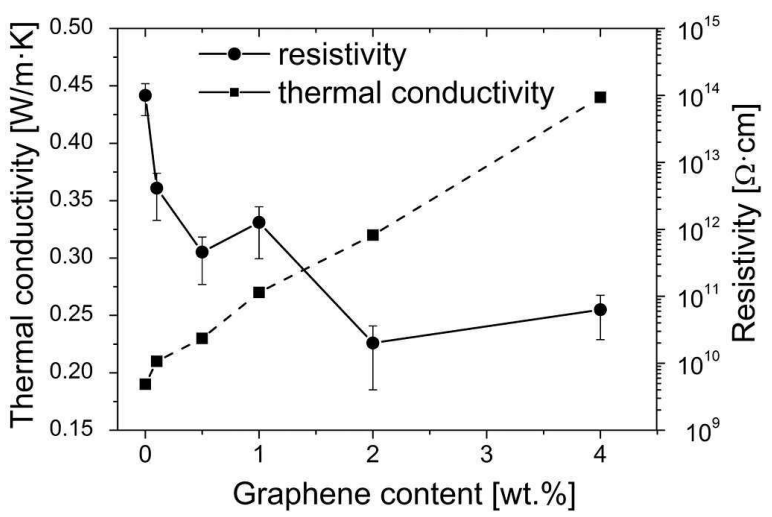

Fig. 4. Thermal conductivity and electrical resistivity of GnP composites.

Four-point probe method gives information about in-plane resistivity. To verify the insulation properties of composites in through-plane direction, the resistivity in sandwich configuration was also measured. Fig. 5 shows the resistivity measured in two ways: by four-point probe method and in sandwich configuration. As seen, for a small amount of graphene in composite (up to 1 wt. \%) the resistivity measured by four-point probe method is lower than that in through-plane direction. This can indicate that the graphene flakes are arranged parallel to the in-plane direction on the sample surface and lead to decrease in resistivity of the samples in this direction.

\subsection{Sedimentation}

Using the results of the research in Hot Disc system, the sedimentation process of graphene particles in epoxy resin can be analyzed. By marking upper and lower side of the cylindrical samples it was possible to test four configurations of the sample set-up which provided information if filler sedimentation occurred in the sample. Nanoparticles fall down under gravity force and it increases

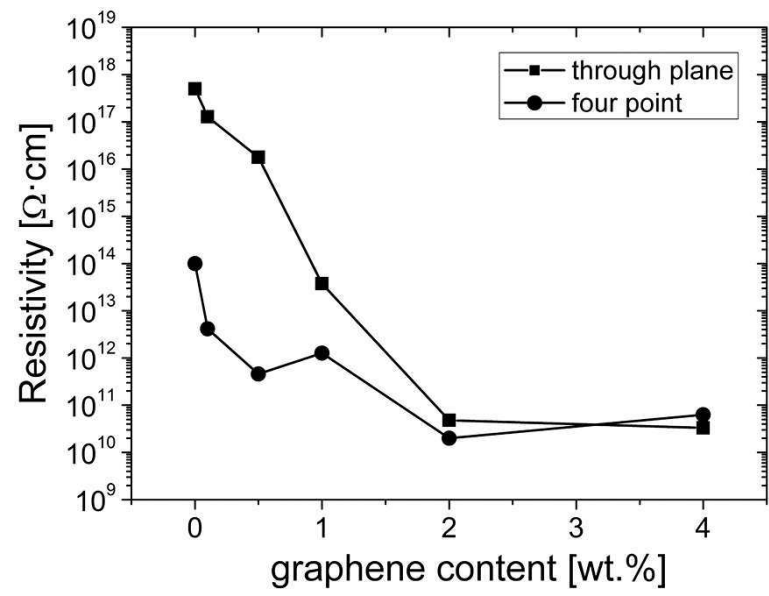

Fig. 5. Resistivity measured in two ways: by four-point probe method and in through-plane configuration.

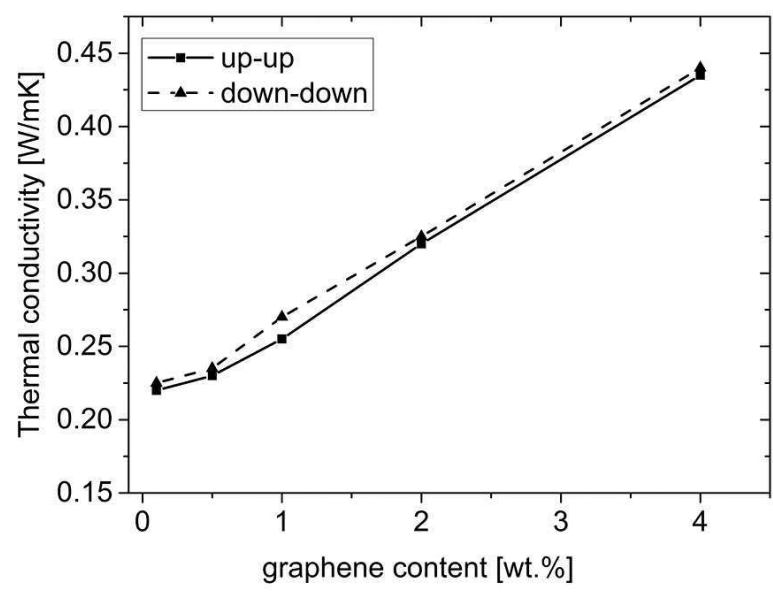

Fig. 6. Sedimentation effect investigated by thermal conductivity measurement.

the thermal conductivity of the composite in the lower part of the composite (down). Higher values of thermal conductivity in down-down configuration than in up-up configuration were expected. This effect may be confirmed by measuring the thermal conductivity in various configurations. Fig. 6 shows that the thermal conductivity in the down-down configuration is a bit higher than in the up-up configuration for each of the filler content. The average value of thermal conductivity was calculated on the basis of four measurement results: down-down, down-up, up-down and up-up. The sedimentation process for graphene flakes in 
epoxy matrix is not as significant as for ceramic particles [29]. It can be described by the high specific surface area of graphene flakes (in our case $\left.\sim 80 \mathrm{~m}^{2} \cdot \mathrm{g}^{-1}\right)$. The buoyancy force acting on the graphene flakes is higher than the force acting, e.g. on ceramics particles. Therefore, graphene flakes are the ideal filler for isotropic applications.

\subsection{Temperature dependence of thermal conductivity}

In the literature [4, 26-28], results of the temperature dependence of thermal conductivity can be found, however, measured only above the room temperature. In order to fully understand the mechanisms which govern the flow of heat it is worth to measure the thermal conductivity at much lower temperatures and compare it with the traditional models. Fig. 7 shows thermal conductivity of selected composites measured as a function of temperature. The measurements have been carried out from $300 \mathrm{~K}$ to $2 \mathrm{~K}$ with different cooling speeds. Firstly, it is worth to say that thermal conductivity results from PPMS correlate well at room temperature with those from the Hot Disc instrument (Fig. 4). Fig. 7 shows a typical parabolic increase of thermal conductivity at the low temperature range, linear dependence at medium temperature range and saturation when approaching room temperature. Therefore, thermal conductivity shows $\mathrm{T}^{2}$ dependence and similarly, graphite also shows $\mathrm{T}^{2.3}$ dependence due to quadratic dispersion of out-of-plane phonon mode at low temperatures [30]. It means that acoustic phonon contribution dominates the thermal transport. At a higher temperature range over $20 \mathrm{~K}$, thermal conductivity of the GnP composites shows either linear temperature dependence which comes from the start of Umklapp process from phonon-phonon scattering [7]. Apart from the above differences, the character of these curves is similar to that of amorphous materials.

\subsection{Modelling}

A very important aspect of the description of thermal properties of isotropic composite containing randomly oriented particles is to create

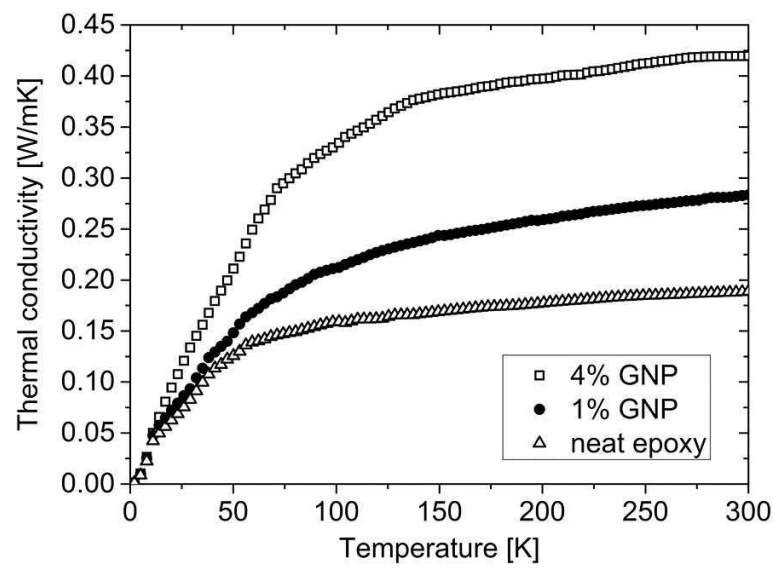

Fig. 7. Temperature dependence of thermal conductivity.

an appropriate model for the thermal conductivity. Recently, Chu et al. [10] proposed a model taking into account the flatness ratio of graphene flakes. The thermal conductivity of GnPs-epoxy composite is expressed as:

$$
\frac{k_{e f f}}{k_{m}}=\frac{3+\frac{2 \cdot \eta^{2} \cdot \Phi}{k_{m} \cdot\left(\frac{2 \cdot R_{k}}{L}+13 \cdot 4 \sqrt{c}\right)}}{3-\eta \cdot \Phi} .
$$

This formula includes all the effects of the thickness c, length $\mathrm{L}$, interfacial thermal resistance $R_{k}$, flatness ratio of $\mathrm{GnP} \eta$ of graphene nanoplatelets (Chu et al. [10]), filler content $\Phi$ and thermal conductivity of the matrix $\mathrm{k}_{\mathrm{m}}$. Here, typical material parameters were used to predict the thermal conductivity of nanocomposite, where $\mathrm{L}=4.5 \mu \mathrm{m}, \mathrm{c}=12 \mathrm{~nm}, \mathrm{k}_{\mathrm{m}}=0.19 \mathrm{~W} \cdot \mathrm{m}^{-1} \cdot \mathrm{K}^{-1}$, $\eta=0.25$ and $R_{k}=3.5 \times 10^{-9} \mathrm{~m}^{2} \cdot \mathrm{K} \cdot \mathrm{W}^{-1}$. Fig. 8 shows the comparison between theoretical calculation and the experimental results. As can be clearly seen, the theoretical results obtained with Chu's model are in good agreement with the presented experimental data. It indicates that the flatness ratio $(\eta)$ has the dominant influence on the thermal conductivity enhancement of GnPs composites.

\section{Conclusions}

In summary, the GnP composites were successfully designed and fabricated in order to obtain the electrically insulating composites with enhanced 


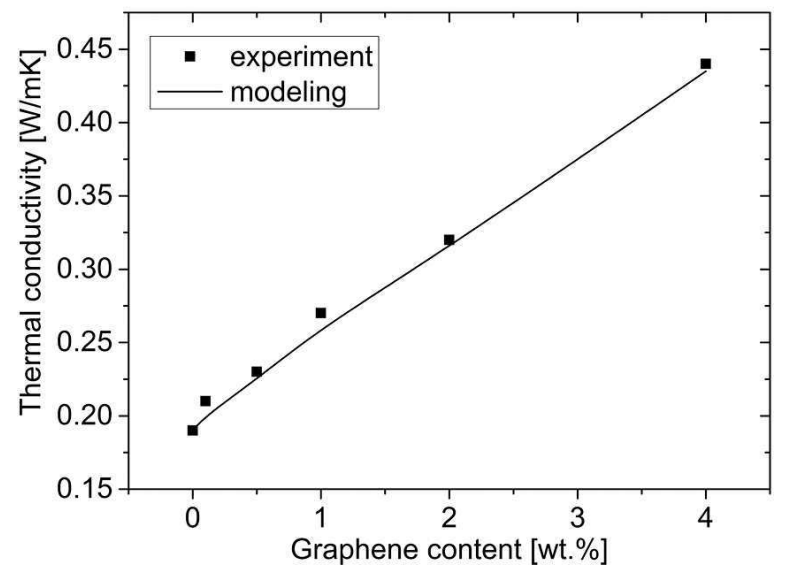

Fig. 8. Chu's model prediction of thermal conductivity as a function of GnPs content compared with the experimental data.

thermal conductivity. The addition of graphene particles into the epoxy resin has improved its thermal conductivity and simultaneously maintained high electrical resistivity as probably the percolation threshold was not exceeded. The addition of only 4 wt. $\%$ of graphene could lead to $132 \%$ increase in thermal conductivity. The excellent agreement between the theoretical model predictions and the experimental data was obtained. The electrical resistivity measured by means of two methods confirmed the insulating properties of the obtained $\mathrm{GnP}$ composites. In order to characterize the thermal properties of the composites, the sedimentation process and temperature dependence of thermal conductivity were examined. The high sheer mixer allowed us to obtain a homogeneous dispersion of the GnP composite samples. The unique structure of the composite makes it a potentially high performance thermally conductive material for the application in electronic devices.

\section{Acknowledgements}

The authors are grateful to Dr. Jacek Nizioł (University of Science and Technology AGH, Department of Solid State Physics in Krakow) for his help in the samples' preparation.

\section{References}

[1] Balandin A., Nat. Mater., 10 (2011), 569.

[2] Novoselov K.S., Geim A.K., Morozov S.V., JiAng D., Zhang Y., Dubonos S.V., GRIGorieva I.V., FIRsov A.A., Science, 306 (2004), 666.
[3] Sartre V., Lallemand M., Appl. Therm. Eng., 21 (2001), 221.

[4] QIAN R., YU J., Wu C., Zhai X., JiAng P., RSC Adv., 3 (2013), 17373.

[5] Chatterjee S., Nafezarefi F., Tai N.H., SchlaGenhauf L., NüEsch F.A., Chu B.T.T., Carbon, 50 (2012), 5380.

[6] Gaska K., Rybak A., Kapusta C., Sekula R., SiWEK A., Polym. Advan. Technol., 26 (2015), 26.

[7] Shahil K.M.F., Balandin A.A., Nano Lett., 12 (2012), 861.

[8] King J.A., Via M.D., Morrison F.A., WiEsE K.R., Beach E.A., Cieslinski M.J., Bogucki G.R., J. Compos. Mater., 46 (2012), 1029.

[9] Zhai W., Shi X., Wang M., Xu Z., Yao J., Song S., WAng Y., ZHAng Q., J. Compos. Mater., 48 (2014), 3727.

[10] Chu K., Jia C., Li W., Appl. Phys. Lett., 101 (2012), 121916.

[11] Song S., Park K., Kim B., Choi Y., Jun G., Lee D., Kong B., PAIK K., JeOn S., Adv. Mater., 25 (2013), 732.

[12] Wang Y., Yu J., Dai W., Song Y., Wang D., Zeng L., JiAng N., Polym. Composite., 36 (2015), 556.

[13] Kausar A., Anwar Z., Muhammad B., Polym.Plast. Technol. Eng, 55 (2016), 1192.

[14] Yu A., Ramesh P., Itkis M.E., Bekyarova E., Haddon R.C., J. Phys. Chem. C, 111 (2007), 7565.

[15] Wei J., Vo T., INAM F., RSC Adv., 5 (2015), 73510.

[16] Wang F., Drzal L.T., QIN Y., Huang Z., J. Mater. Sci., 50 (2015), 1082.

[17] Chatterjee S., Wang J.W., Kuo W.S., Tai N.H., SAlzMAnN C., Li W.L., Hollertz R., NÜESCH F.A., Chu B.T.T., Chem. Phys. Lett., 531 (2012), 6.

[18] Balandin A.A., Ghosh S., Bao W., Calizo I., Teweldebrhan D., Miao F., LaU C.N., Nano Lett., 8 (2008), 902.

[19] NiKa D., Pokatilov E., Askerov A., BALANDIN A., Phys. Rev. B, 79 (2009), 155413.

[20] Ghosh S., Bao W., NiKa D.L., Subrina S., Pokatilov E.P., Lau C.N., Balandin A.A., Nat. Mater, 9 (2010), 555.

[21] Paton K.R., VArRla E., Backes C., SMith R.J., Khan U., O’Neill A., Boland C., Lotya M., Istrate O.M., King P., Higgins T., Barwich S., May P., Puczkarski P., Ahmed I., Moebius M., Pettersson H., Long E., Coelho J., O’Brien S.E., McGuire E.K., SAnchez B.M., DuesberG G.S., McEvoy N., Pennycook T.J., Downing C., Crossley A., Nicolosi V., Coleman J.N., Nat. Mater., 13 (2014), 624.

[22] Yi M., Shen Z., J. Mater. Chem. A, 3 (2015), 11700.

[23] LiU L., Shen Z., Yi M., Zhang X., Ma S., RSC Adv., 4 (2014), 36464.

[24] Song N., Jia J., Wang W., GaO Y., ZhaO Y., Chen Y., Chem. Eng. J., 298 (2016), 198.

[25] Wang S., TAmbraparni M., QIU J., Tipton J., DEAN D., Macromolecules, 42 (2009), 5251. 
[26] Park W., Hu J., Jauregui L.A., Ruan X., Chen Y.P., Appl. Phys. Lett., 104 (2014), 113101.

[27] Ramirez C., Figueiredo F.M., Miranzo P., Poza P., Osendi M.I., Carbon, 50 (2012), 3607.

[28] Gu J., Xie C., Li H., Dang J., Geng W., Zhang Q., Polym. Composite, 35 (2013), 1087.
[29] Sun Y.-H., Xiong W.-H., Li C.-H., T. Nonferr. Metal. Soc., 20 (2010), 624.

[30] Choi S., Kim J., Compos. Part B-Eng., 51 (2013), 140 Received 2016-10-04 Accepted 2017-01-16 